\title{
Infección urinaria en pediatría: controversias
}

\author{
Felipe Cavagnaro
}

\section{Urinary tract infection in pediatrics: controversies}

Urinary tract infection is a frequent event in children. The correct diagnosis, management and study has been involved in major controversies in the last decade as a result of better design of scientific studies and evidencebased medicine in this area. A significant amount of our knowledge must be subjected to critical analysis because of new information available. This article describe some of the controversies in pediatric urinary tract infection that should be considered by the health team, highlighting the relevance of sample collection and the significant number of bacterial colonies, the type and duration of antibiotic treatment, the prescription of antibiotic prophylaxis and the required imaging study.

Key words: Urinary tract infection, pyelonephritis, vesicoureteral reflux, prophylaxis.

Palabras clave: Infección urinaria, pielonefritis, reflujo vesicoureteral, profilaxis.

\section{Introducción}

$\mathrm{E}$ n el año 2005 publiqué en esta revista un artículo de revisión sobre infección del tracto urinario (ITU) en pediatría ${ }^{1}$, en el que vertí la mayoría de los conceptos consensuados, hasta ese momento, sobre los distintos aspectos clínicos de este diagnóstico. Desde ese entonces, y apoyado en el mejor diseño de los trabajos científicos y de la medicina basada en evidencias, muchos de estos conceptos han sido modificados, relativizados o al menos debatidos en su real importancia o trascendencia. El presente artículo pretende actualizar, en algunos aspectos, la información disponible sobre ITU y sus principales complicaciones, en los que han aparecido controversias de enorme significado clínico, y que deben ser conocidas y sopesadas a la hora de tomar decisiones de estudio y tratamiento en este grupo de pacientes, evitando inducir al lector, a la manera de una guía clínica, hacia una u otra alternativa. Como veremos, aún hay bastante camino por recorrer, y muchas preguntas por responder. Algunos avances recientes en diagnóstico, estudio y tratamiento, en etapa de validación clínica, no serán revisados en este artículo, ya que aún no están en el rango de controversia en relación a lo que comúnmente se realiza.

\section{Controversia 1. De la toma de examen de orina y recuento bacteriano}

Nuestro conocimiento "fundacional" del número de unidades formadoras de colonias (ufc) necesarias para confirmar el diagnóstico de ITU en relación al método de toma de muestra proviene de un estudio publicado hace más de medio siglo en un grupo de mujeres embarazadas sintomáticas ${ }^{2}$. De este estudio se dedujo que el recuento de ufc era significativo para ITU si alcanzaba un número $\geq 100.000 / \mathrm{ml}$ de orina de un mismo agente patógeno en muestras tomadas de "segundo chorro", aunque razones técnicas impedían realizar conteos mayores. Recientemente, se demostró que, en población pediátrica, este número originaba al menos $7 \%$ de falsos positivos, lo que se reducía enormemente, sin perder sensibilidad, si este número se elevaba a $1.000 .000 \mathrm{ufc} / \mathrm{ml}^{3}$. En el caso de muestras tomadas por cateterización trans-uretral o "sondeo" vesical, hay grandes variaciones en lo que se considera significativo, yendo desde $>1.000 \mathrm{ufc} / \mathrm{ml}$ en niñitas y niños circuncidados y $>100.000 \mathrm{ufc} / \mathrm{ml}$ en los no circuncidados ${ }^{4}, \geq 10.000 \mathrm{ufc} / \mathrm{ml}$ en lo aceptado por nuestra Sociedad Chilena de Infectología ${ }^{5}$ ( $\mathrm{mi}$ artículo $^{1}$ ), hasta un valor intermedio de $\geq 50.000 \mathrm{ufc} / \mathrm{ml}$ utilizado en guías clínicas y artículos de investigación clínica de trascendencia ${ }^{6,7}$. Se considera que la uretra distal y el área periuretral están usualmente colonizadas con la misma bacteria que puede causar la ITU, por lo que no es raro un bajo recuento de colonias en muestras de orina por cateterización trans-uretral en pacientes con orina vesical sin bacterias. En el caso de la punción supra-púbica, parece haber más consenso en considerar cualquier recuento como significativo para diagnosticar $\mathrm{ITU}^{5,8}$, aunque otras guías sugieren diferenciar en el tipo de bacterias, exigiendo $>100^{9}$ ó $>1.000 \mathrm{ufc} / \mathrm{ml}^{4}$ si se trata de una cocácea grampositiva. Es importante tener en cuenta que el resultado positivo o negativo de un urocultivo es absolutamente operacional y no absoluto, siendo muy importante, en este sentido, el tiempo de incubación intravesical de las bacterias como determinante de la magnitud del recuento de colonias.

Todos los métodos de recolección de muestra de orina
Facultad de Medicina Clínica Alemana-Universidad del Desarrollo.

Departamento de Pediatría.

Conflictos de interés: no hay.

Recibido: 27 de febrero de 2012 Aceptado: 8 de mayo de 2012

Correspondencia a: Felipe Cavagnaro Santa María fcavagnaro@alemana.cl 
pueden tener contaminaciones (falsos positivos), siendo menor con la punción supra-púbica, un poco mayor (y similar entre ellas) en cateterismo vesical y "segundo chorro" 10 , e inaceptablemente alta por bolsa recolectora, en cuyo caso se puede encontrar contaminación hasta en $86 \%$ de las muestras ${ }^{7,11}$. Ahora bien, visto de otro modo, un urocultivo negativo de una muestra tomada por bolsa recolectora tiene un excelente valor predictor negativo para ITU. En forma práctica, cuando debe iniciarse pronto una conducta terapéutica en un niño/a con sospecha de ITU, se recomiendan los métodos "invasores" en los niños sin control voluntario del esfínter urinario, y el "segundo chorro" en aquellos con continencia urinaria.

La aparición de más de una especie microbiana en el cultivo sugiere fuertemente una contaminación, aunque no debe descartarse de plano que pueda corresponder a una ITU real por más de un tipo de bacteria ${ }^{12}$. Por otro lado, un urocultivo negativo, en presencia de síntomas urinarios, puede reflejar nuestra incapacidad de detectar, con el medio de cultivo tradicional, microorganismos patógenos menos frecuentes ${ }^{13}$.

Otro punto en conflicto es el concepto de bacteriuria asintomática, definida como un recuento significativo de bacterias en orina (dependiendo del método empleado de recolección) en ausencia de síntomas detectables; la presencia de piuria significativa concomitante sería la regla para algunos ${ }^{14}$ y descartaría este diagnóstico para otros $^{15}$. En lo personal, esta última conducta me parece más adecuada ya que la presencia de piuria refleja inflamación en algún punto de la vía urinaria.

La utilidad de un análisis de orina complementario al urocultivo en el diagnóstico de ITU es significativo y es parte de los consensos publicados s $^{7,16,17}$.

\section{Controversia 2. De la decisión antimicrobiana y los plazos de tratamiento}

Las metas del tratamiento de una ITU son eliminar la infección aguda y sus síntomas, prevenir sus complicaciones y reducir la probabilidad de un daño renal a futuro. Para ello, es de suma importancia conocer cuatro factores que influyen en la elección del antimicrobiano y la duración del tratamiento: el tipo de bacteria y su susceptibilidad in vitro, la ubicación de la ITU (alta o baja), el contexto clínico (tolerancia, gravedad, etc.) y la edad del paciente. Normalmente, el inicio de la antibioterapia ocurre previo a conocerse el resultado del urocultivo, por lo que la elección del fármaco suele tener una base empírica y basada en la epidemiología local. Parece claro que, dado que sobre $90 \%$ de los agentes uropatógenos son bacterias entéricas gramnegativas, el tratamiento inicial debe incorporar, al menos, un antimicrobiano activo sobre estas bacterias (usualmente una cefalosporina o un amino- glucósido), adicionando ampicilina en casos de niños bajo 2 meses de edad, en que la incidencia de Enterococcus sp es mayor que en otras edades ${ }^{7,15-17}$. La epidemiología institucional nos puede ayudar en la elección del antimicrobiano, pero debe tenerse presente el hecho de que la resistencia a antimicrobianos usuales está en constante aumento. Por esta razón, recomiendo revisar y conocer la susceptibilidad in vitro de los principales agentes uropatógenos del área o centro de salud donde trabajen, más que seguir las sugerencias de publicaciones extranjeras. El uso de fluoroquinolonas, tan controvertido en niños por el riesgo de toxicidad articular, constituye un potencial agente antimicrobiano de primera línea en pielonefritis e ITUs complicadas, en situaciones en que los antimicrobianos usualmente recomendados no son apropiados en base a su susceptibilidad in vitro o antecedentes de eventos $\operatorname{adversos}^{18}$. La Academia Americana de Pediatría (AAP) apoya actualmente el uso de ciprofloxacina como terapia oral de las ITUs causadas por Pseudomonas aeruginosa u otras bacterias gramnegativas multi-resistentes, en niños entre 1 y 17 años ${ }^{19}$.

Tradicionalmente, la duración del tratamiento de una ITU dependerá si es alta o baja (ver Controversia 6). Para las ITUs altas (o pielonefritis, para efectos clínicos), la duración sugerida, especialmente en niños bajo 2 años de edad, es de 7 a 14 días, pudiendo ser en base a antimicrobianos orales en ausencia de sepsis clínica y/o mayores de 2-3 meses, o comenzando con un fármaco parenteral de amplio espectro (p. ej.: ceftriaxona o aminoglucósidos i.v.) para luego completar este tiempo con una alternativa oral (p. ej.; cefalosporina de $2^{\mathrm{a}}$ ó $3^{\mathrm{a}}$ generación $)^{7,20}$. No existe información que permita comparar la eficacia de pautas de 7-10 días con las de mayor duración ${ }^{16,21}$. Tampoco hay trabajos bien diseñados en niños con tratamientos acortados para pielonefritis, pero hay evidencias que tratamientos de ITUs febriles durante 1 a 3 días son menos exitosos que tratamientos más prolongados ${ }^{7}$. En una reciente revisión Cochrane, no se observaron diferencias en la generación de cicatrices renales o persistencia de la fiebre entre pacientes tratados con antimicrobianos orales por 10 a 14 días y otros tratados con antimicrobianos i.v. por tres días, seguidos de 10 días de tratamiento oral ${ }^{22}$. Es necesario aclarar que en muchos de los trabajos analizados se excluían paciente con cicatrices previas o malformaciones nefro-urológicas, en los que la conducta podría ser distinta. En las ITUs bajas o cistitis, la duración clásica sugerida para un tratamiento antimicrobiano oral es de 7 días, aunque recientes revisiones sugieren que sería suficiente con 2 a 5 días, no habiendo evidencia de ventajas con terapias más prolongadas ${ }^{15,21}$. Con fines de simplificar conductas, Saadeh y Mattoo plantean que niños bajo dos años de edad, con sospecha de ITU, deben ser tratados como una pielonefritis; en cambio, un tratamiento más basado en la clínica puede ser utilizado 


\section{Infectología al Día}

en niños mayores ${ }^{15}$. En casos de ITUs complicadas, es decir, asociadas a alteraciones anatómicas subyacentes o con riesgo de abscedación, la terapia puede ser más prolongada, y por al menos cinco días después de que caiga la fiebre ${ }^{15,23}$.

La bacteriuria asintomática no debe tratarse dado que no se ha demostrado un riesgo aumentado de infecciones renales y/o daño renal cicatricial ${ }^{15}$, a excepción de que se presente en pacientes adolescentes cursando un embarazo ${ }^{24} \mathrm{o}$ en pacientes en los que se realizará un procedimiento invasor sobre la vía urinaria ${ }^{25}$.

Aunque hay estudios que sugieren que una ITU tratada precozmente $(<24 \mathrm{~h}$ de inicio de síntomas) no tiene mayor incidencia de cicatrices renales que una tratada más tardíamente ${ }^{26,27}$, el tratamiento no debe retrasarse en un niño enfermo, ya que pueden ocurrir otras complicaciones, como urosepsis o abscesos renales ${ }^{15}$.

\section{Controversia 3. De la indicación de hospitalización}

Las indicaciones más clásicas de hospitalización de un niño con ITU febril son: lactante bajo 6 a 8 semanas de vida, urosepsis clínica, evidencia de laboratorio de bacteriemia, intolerancia oral, paciente inmunocomprometido, antecedentes de uropatía o nefropatía grave, riesgo de pérdida del control ambulatorio o caso social, y falla en la respuesta a tratamiento ambulatorio ${ }^{15,20}$. El hecho de hospitalizar a lactantes bajo 2 meses de edad, con ITU febril, obedece al elevado riesgo de bacteriemia $(10 \%)$ en ellos ${ }^{26}$, aunque la progresión de esta enfermedad a etapas más graves parece ser poco común ${ }^{28}$. Experiencias clínicas han demostrado que el manejo de pacientes cada vez más pequeños ( $>$ a 2 meses) en forma ambulatoria, con terapia oral, es perfectamente posible y no adiciona mayor riesgo de daño renal a futuro ${ }^{22}$. Por otro lado, la utilización de una dosis parenteral de cefalosporina de $3^{\mathrm{a}}$ generación, de acción prolongada como ceftriaxona, o de un aminoglucósido como amikacina o gentamicina, permiten enviar a domicilio a pacientes con ITUs con mala tolerancia oral, a la espera de mejoría de sus síntomas dentro de las 24 a $48 \mathrm{~h}$ siguientes, para iniciar terapia $\operatorname{oral}^{7,20}$. Por último, la falla en la respuesta a tratamiento ambulatorio es algo mal definido, ya que generalmente se centra en la duración de los síntomas, especialmente la fiebre. En este sentido, Bachur demostró que en un grupo de niños con primera ITU febril y sin problemas urológicos o inmunodeficiencias conocidos, la fiebre cayó en las primeras $48 \mathrm{~h}$ en $89 \%$ de los pacientes (respondedores). El otro $11 \%$ (no respondedores) tuvo una similar frecuencia de bacteriemias, hidronefrosis ecográficas, abscesos renales y reflujo vesico-ureteral (RVU) significativo que los respondedores. Todos los urocultivos controles fueron negativos $^{29}$.

\section{Controversia 4. De la profilaxis} antimicrobiana

El hecho de que la profilaxis antimicrobiana previene o reduce el número de ITUs es una idea bastante generalizada. Como consecuencia de esto, además, prevendría el desarrollo de nuevas cicatrices renales, particularmente en aquellos niños con RVU, reduciendo así el riesgo de progresión hacia la insuficiencia renal crónica ${ }^{7}$. En el sentido opuesto, la profilaxis antimicrobiana puede exponer al niño a reacciones adversas del medicamento, y a la selección de cepas bacterianas más resistentes. Una revisión sistemática reciente de pacientes pediátricos con una primera ITU mostró que $57 \%$ tuvo cambios cintigráficos consistentes con pielonefritis aguda, y 15\% quedó con evidencias de cicatriz renal en una evaluación tardía, siendo los niños con RVU, especialmente los de alto grado, los de mayor porcentaje de lesiones crónicas ${ }^{30}$. De este modo, parece razonable identificar una manera de prevenir este daño renal, el cual parece ser acumulativo en la medida de que aumenta el número de pielonefritis agudas ${ }^{31}$. Numerosos estudios realizados en los últimos años no han demostrado utilidad en el uso de profilaxis antimicrobiana para prevenir ITUs ${ }^{32,33}$, al punto que algunas importantes guías clínicas, como las del Instituto Nacional para la Salud y Excelencia Clínica (NICE, por sus iniciales en inglés) del Reino Unido, desaconsejan su uso rutinario en niños con primera ITU ${ }^{17}$. Una reciente revisión sistemática Cochrane sobre estudios que comparaban profilaxis antimicrobiana con placebo o ningún tratamiento, mostró que los antimicrobianos no parecieron reducir el riesgo de presentar una ITU sintomática ${ }^{34}$. Sin embargo, cuando se evaluaron los efectos de los antimicrobianos en estudios con bajo riesgo de sesgo, hubo una reducción estadísticamente significativa. El efecto fue similar en los niños con RVU en comparación con los niños sin RVU. No hubo consistencia en la aparición de eventos adversos, pero algunos estudios mostraron resistencia al antimicrobiano en los grupos de tratamiento activo. Por lo anterior, concluyen que el tratamiento prolongado con antimicrobianos profilácticos parece reducir el riesgo de repetición de la ITU sintomática en los niños susceptibles, pero el beneficio es pequeño y debe considerarse junto con el mayor riesgo de resistencia microbiana $^{34}$. En un comentario editorial, Montini y Hewitt concluyeron, un poco antes, que la profilaxis antimicrobiana no está indicada para niños luego de una primera ITU febril si no tienen RVU o éste es de grado $\leq 2$; en caso de RVU mayores aún no hay una conclusión definitiva ${ }^{35}$.

\section{Controversia 5. Del uso de otras medidas preventivas}

Diversas medidas preventivas han sido claramente demostradas como útiles en ITU pediátrica, destacando 
el solucionar y evitar la estitiquez, el buen aseo génitoanal y la frecuente eliminación de orina, evitando así su retención vesical ${ }^{36}$. Otras medidas más controversiales se discuten a continuación:

- La circuncisión. La relación entre circuncisión en varones e ITU ha sido largamente debatida. Actualmente, se considera que la circuncisión reduce la tasa de ITU en lactantes bajo 6 meses de edad, en alrededor de 10 veces. En edades posteriores no parece tener mayor beneficio ${ }^{37}$. En niños sanos, el riesgo de ITU es de alrededor de $1 \%$, lo que significa que hay que circuncidar a 111 niños para evitar una ITU, pero este número disminuye considerablemente si el niño tiene historia de ITUs recurrentes o RVU de alto grado ${ }^{38}$. Estos datos han llevado a considerar en los algoritmos terapéuticos que el lactante varón con fiebre sin foco tenga mayor riesgo de ITU si no está circuncidado ${ }^{7}$. Si bien es actualmente planteable la circuncisión en niños con ITUs recurrentes y/o con malformaciones genitourinarias $/ \mathrm{RVU}^{38}$, la discusión sobre circuncidar o no a los neonatos sanos en forma profiláctica es aún muy debatida ${ }^{39,40}$.

- El jugo de cranberry (arándano rojo o agrio). Existen algunas evidencias que el jugo de cranberry puede disminuir el número de ITUs sintomáticas en mujeres, particularmente en aquellas con ITUs recurrentes ${ }^{41}$. En el caso de niños, un reciente y bien diseñado estudio finés no mostró una significativa reducción en el número de pacientes con recurrencias de ITUs, pero fue efectivo en reducir el número total de recurrencias ${ }^{42}$. Quizás uno de los problemas de esta alternativa es que el jugo de cranberry es poco aceptado por niños por períodos prolongados de tiempo.

- La lactancia materna. Si bien no existen estudios bien diseñados que investiguen el efecto protector de la leche materna en prevenir ITUs, hay algunas evidencias que lo avalan. Un estudio sueco observó que la lactancia al pecho exclusiva disminuyó significativamente la tasa de recurrencias de ITUs, efecto que se mantenía al menos hasta los 7 meses si continuaba lactando ${ }^{43}$. En prematuros, el efecto protector de la leche materna también fue observado ${ }^{44}$. El mecanismo defensivo que produce la leche materna en el tracto urinario aún no ha sido aclarado.

Los probióticos. Constituidos por microorganismos vivientes potencialmente benéficos para nuestro organismo, los probióticos han mostrado gran utilidad en prevenir infecciones gastrointestinales ${ }^{45}$. En relación a prevenir ITUs, su utilidad ha sido especialmente documentada en mujeres y con ciertas cepas de Lactobacillus $^{46}$. En el caso de niños, algunos ensayos terapéuticos han tenido resultados favorables en relación disminuir las recurrencias de ITUs, pero no logran tener una utilidad concluyente ${ }^{47,48}$. En un futuro, el beneficio de los probióticos puede relacionarse con el uso de agentes microbiológicos específicos que puedan eliminar a los uropatógenos de alta virulencia de su reservorio en el tracto gastrointestinal ${ }^{49,50}$.

\section{Controversia 6. De la ubicación de la infección urinaria}

Es importante saber qué estructuras de la vía urinaria están afectadas por la infección, y en qué grado. Básicamente, la pregunta se traslada a demostrar si hay o no compromiso del parénquima renal (pielonefritis aguda), ya que este dato puede condicionar un estudio y/o tratamiento diferenciado (Controversias 2 y 7), e implica un riesgo distinto de daño renal futuro. Tradicionalmente, el cuadro de fiebre y compromiso del estado general en un niño con molestias urinarias y, por supuesto, con urocultivo positivo, se considera clínicamente sinónimo de pielonefritis. Este enfoque parece no ser totalmente correcto cuando se correlaciona con el actual estándar de oro del compromiso renal: la cintigrafía renal con Tc99 DMSA realizada en fase aguda de la ITU febril, en que en sólo $60 \%$ se sugiere compromiso del parénquima renal ${ }^{51}$. Por otro lado, la evaluación concomitante con exámenes de sangre que reflejen inflamación aguda como leucocitosis, elevación de la velocidad de eritrosedimentación y de la proteína $\mathrm{C}$ reactiva, aumentan la sensibilidad de diagnóstico pero con baja especificidad ${ }^{52}$. En este grupo de exámenes, la procalcitonina pudiera constituirse en un marcador más específico de compromiso renal ${ }^{38,53,54}$. Una reciente experiencia publicada por nuestro grupo demostró que la ecografía renal con efecto Doppler asociado aumentaba fuertemente, y de manera, poco invasora, la posibilidad de localizar correctamente el nivel de inflamación ${ }^{55}$. La TAC y la resonancia magnética mostraron similar sensibilidad y confiabilidad en el diagnóstico de pielonefritis aguda que la cintigrafía renal con DMSA, y todas un poco superiores a la ecografía renal con Doppler ${ }^{56}$.

La presencia de cilindros piocitarios en el sedimento urinario son altamente sugerentes de pielonefritis pero son poco frecuentes de observar ya que suelen desintegrarse con la centrifugación de la muestra de orina. Otros elementos de laboratorio, como bacterias recubiertas de anticuerpos, citoquinas urinarias y enzimas tubulares renales son aún de difícil acceso clínico rutinario, por lo que no son considerados de utilidad práctica por el momento ${ }^{57}$.

\section{Controversia 7. Del estudio imagenológico de la vía urinaria}

La importancia de un estudio imagenológico del riñón y la vía urinaria es el conocer eventuales condiciones anatómicas y/o fisiológicas del paciente que expliquen la 
ITU o aumenten el riesgo de recurrencias de ITU, y por ende, el de daño renal crónico.

El ultrasonido renal y vesical, técnica no invasora y no irradiante, es un estudio muy útil para evaluar el parénquima y tamaño renal, malformaciones renales congénitas con y sin hidronefrosis, abscesos renales y, en algunas ocasiones, presencia de litiasis y/o nefrocalcinosis. En el contexto de una ITU, la ecografía descubre pocas situaciones (1 a $2 \%$ ) que requieran una acción médica específica ${ }^{7}$, probablemente en relación a que la generalización de la ecografía obstétrica detecta la gran mayoría de las malformaciones nefro-urinarias. Pese a eso, en mi experiencia he visto pacientes con graves alteraciones congénitas del riñón y vías urinarias cuyas ecografías prenatales fueron informadas como "normales", por lo que concuerdo absolutamente con las recomendaciones de la AAP y NICE en el sentido de que este estudio debiera ser realizado en todo niños con ITU febril dentro de las primeras $48 \mathrm{~h}^{7,17}$.

La uretrocistografía, estudio que requiere cateterización vesical e irradación, es el mejor examen conocido para demostrar y graduar un RVU, siendo este último la principal "causa" demostrable de ITUs altas y recurrentes. Sin embargo, está claro que en un gran porcentaje de los niños que tiene pielonefritis ( $>60 \%$ ) no se puede demostrar RVU ${ }^{58}$. Por otro lado, algunos estudios han sugerido que la profilaxis antimicrobiana no confiere el beneficio deseado de prevenir ITUs febriles recurrentes (Controversia 4). De este modo, la racionalidad de realizar una uretrocistografía rutinaria en niños después de una primera ITU febril es, al menos, cuestionable. La AAP sugiere no realizar este examen en forma rutinaria después de la primera ITU febril, a menos que la ecografía demuestre hidronefrosis, cicatrices renales u otros hallazgos que sugieran RVU de alto grado o uropatía obstructiva, como así en las recurrencias de ITUs febriles ${ }^{7}$. Para ser más selectivos con este examen, el grupo británico propuso realizar una uretrocistografía exclusivamente en niños bajo 6 meses de edad con una ITU atípica (bacteria no Escherichia coli en el urocultivo, seriamente enfermo, chorro urinario débil, masa abdominal o vesical, elevación de la creatininemia, falla de respuesta al tratamiento antimicrobiano a las $48 \mathrm{~h}$ ) o una ITU recurrente (dos o más episodios de ITU alta, o un episodio de ITU alta más uno o más episodios de ITU bajas, o tres o más episodios de ITUs bajas) ${ }^{17}$. La uretrocistografía también podría ser solicitada si hay historia familiar de RVU ${ }^{59}$.

La cintigrafía renal con DMSA requiere acceso venoso y produce baja radiación. Es la mejor manera, por ahora, de diagnosticar una ITU con compromiso renal, pero no diferencia adecuadamente lesiones agudas de crónicas. Además, su resultado afecta con muy baja frecuencia el manejo clínico agudo, por lo que no estaría indicado en la evaluación rutinaria de niños con una primera
ITU febril ${ }^{7}$. Las normas británicas sugieren realizar una cintigrafía con DMSA en aquellos niños bajo 3 años de edad con ITUs graves o atípicas, y a toda edad en ITUs recurrentes ${ }^{17}$. Una propuesta alternativa a la realización de la uretrocistografía en primera instancia sería comenzar con una ecografía y una cintigrafía renal DMSA ("top down approach"). Si alguno de estos dos exámenes estuviera alterado, se debería continuar el estudio con una uretrocistografía ${ }^{60}$. En nuestro grupo de trabajo, la mayor indicación de una cintigrafía renal con DMSA en la fase aguda es en aquellos pacientes con fiebre de origen desconocido en los que se sospecha una pielonefritis, pero no se cuenta, por diversas razones, con un urocultivo positivo que la demuestre.

Como pueden observar los lectores, aún no hay un consenso amplio en relación al estudio imagenológico a solicitar en un niño con ITU, pero la ecografía renal y de vías urinarias parece estar ampliamente aceptada por todos.

\section{Controversia 8. De la derivación al especialista}

En principio, las ITUs en niños debieran ser evaluadas y tratadas por pediatras generales. La decisión de derivar un paciente con antecedentes de ITU a un especialista (nefrólogo, urólogo) tiene, en parte, relación con la capacidad resolutiva del médico tratante, de los recursos disponibles para su estudio y seguimiento, y con algunas características de los pacientes que los hacen con mayor riesgo de complicaciones o daño renal futuro. Deben destacarse, entre éstas, la presencia de malformaciones nefro-urinarias, RVU de alto grado, ITUs recurrentes, sospecha de vejiga neurogénica, asociación con litiasis, compromiso de la función renal y/o hipertensión arterial, y evidencias de cicatrices renales.

Como suele suceder en muchas patologías pediátricas, ocasionalmente la angustia parental puede ser una causa atendible de derivación al especialista.

\section{Controversia 9. Del seguimiento}

En forma tradicional, el formato de seguimiento en los niños con antecedentes de ITU consistía en urocultivos periódicos en pacientes asintomáticos, como una manera de "adelantarse a la aparición de síntomas". Actualmente, no se considera necesario repetir el urocultivo luego del tratamiento si el paciente muestra una buena respuesta clínica $^{61}$. Tampoco es necesario demostrar la esterilización de la orina intra-tratamiento si la respuesta clínica es adecuada ${ }^{62}$.

Dado que un tratamiento precoz puede limitar un eventual daño renal crónico, está fuertemente recomendado 
instruir a los padres de niños con ITUs febriles en buscar una evaluación médica y tomar un urocultivo en forma confiable, acompañado de un análisis de orina, dentro de las $48 \mathrm{~h}$ de iniciado un episodio febril sin sintomatología de focalización clara ${ }^{7}$.

\section{Conclusiones}

Como menciona el doctor Kjell Tullus en un reciente comentario editorial en una prestigiosa revista científica "la infección urinaria en niños es uno de los, si no el más controvertido campo de la medicina pediátrica"63. La incorporación de nuevas y mejores técnicas diagnósticas, de agentes antimicrobianos de acción más prolongada y con menor toxicidad, y una mayor racionalidad en la forma en que hacemos el seguimiento de estos niños, todas materias de análisis por medicina basada en evidencias, han hecho que muchos dogmas en relación a esta patología, varios de los cuales publiqué en mi anterior artículo ${ }^{1}$, se hayan derrumbado o estén, al menos, en franca discusión. El número significativo de colonias de bacterias patógenas en orina, los tiempos adecuados de tratamiento antimicrobiano, el momento y tipo de estudio de imágenes y el uso de profilaxis en estos pacientes, entre otras controversias, siguen siendo materia de debate clínico. De hecho, en un plazo mediato, algunos estudios clínicos randomizados, actualmente en curso, debieran aportar más claridad en puntos controversiales de este tema y estimulen a redireccionar la forma en que cuidamos de estos pacientes ${ }^{7}$.

\section{Resumen}

La infección urinaria es un evento frecuente en pediatría. Su correcto diagnóstico, manejo y estudio ha estado envuelto en grandes controversias en la última década a raíz del mejor diseño de los trabajos científicos y de la medicina basada en evidencias en esta área. Un importante volumen de nuestro conocimiento al respecto debe ser sometido a un análisis crítico a la luz de la nueva información existente. El presente artículo expone algunas de las controversias sobre infección urinaria en pediatría que deben ser tomadas en cuenta por el equipo de salud que cuida de estos pacientes, entre las que destacan la importancia de la toma de muestra y el número de colonias bacterianas que consideramos significativas, el tipo y duración del tratamiento antimicrobiano, la indicación de profilaxis antimicrobiana y el correcto estudio imageneológico de ellos.

\section{Referencias bibliográficas}

1.- Cavagnaro F. Infección urinaria en la infancia. Rev Chilena Infectol 2005; 22: 161-8.

2.- Kass E H. Bacteriuria and the diagnosis of infections of the urinary tract; with observations on the use of methionine as a urinary antiseptic. Arch Intern Med. 1957; 100: 709-14.

3.- Coulthard M, Kalra M, Lambert H, Nelson A, Smith T, Perry J. Redefining urinary tract infections by bacterial colony counts. Pediatrics 2010; 125: 335-41

4.- Feld L, Matoo T. Urinary tract infections and vesico-ureteral reflux in infants and children. Pediatr Rev 2010, 31: 451-63.

5.- Comité de Microbiología Clínica, Sociedad Chilena de Infectología. Recomendaciones para el diagnóstico microbiológico de la infección urinaria. Rev Chilena Infectol 2001; 18: 57-63.

6.- Hoberman A, Wald E, Reynolds E, Penchansky L, Charron M. Is urine culture necessary to rule out urinary tract infection in young febrile children? Pediatr Infect Dis 1996; 15: 304-9.

7.- Subcommittee on Urinary Tract Infection, Steering Committee on Quality Improvement and Management. Urinary tract infection: Clinical practice guidelines for the diagnosis and management of the initial UTI in febrile infants and children 2 to 24 months. Pediatrics
2011; 128: 595-610.

8.- Williams G, Craig J. Chapter 35: Diagnosis and management of urinary tract infections. Geary D, Schaefer F, editors. Comprehensive Pediatric Nephrology. $1^{\text {st }}$ edition, Philadelphia, MosbyElsevier; 2008, p. 539-48.

9.- Zorc J, Kiddoo D, Shaw K, Shaw K. Diagnosis and management of pediatric urinary tract infections. Clin Microbiol Rev 2005; 18 : 417-22.

10.- Karacan C, Erkek N, Senel S, Akin Gunduz S, Catli G, Tavil B. Evaluation of urine collection methods for the diagnosis of urinary tract infection in children. Med Princ Pract 2012; 19: 188-91.

11.- Leong YV, Tan KW. Bladder aspiration for diagnosis of urinary tract infection in infants and young children. J Singapore Paediatr Soc 1976; 18: 43-7.

12.- Chon C, Lai F, Dairiki-Shortlife L. Pediatric urinary tract infections. Pediatr Clin North Am 2001; 48: 1441-59.

13.- Bensman A, Dunand O, Ulinski T. Chapter 54: Urinary tract infections. Avner E, Harmon W, Niaudet P, Yoshikawa N, editors. Pediatric Nephrology, sixth edition Springer Verlag, Heidelberg; 2009, p. 1299-310.

14.- http://www.topalbertadoctors.org/informed practice/clinical_practice_guidelines/ complete $\% 20$ set/Urinary\%20Tract $\% 20$ Infection/urinary_tract_infection_guideline.pdf
15.- Saadeh S, Mattoo T. Managing urinary tract infections. Pediatr Nephrol 2011; 26: 1967-76.

16.- Ochoa C, Málaga S y Panel de expertos de la Conferencia de Consenso y Grupo Investigador de la Conferencia de Consenso. Recomendaciones de la Conferencia de Consenso "Manejo diagnóstico y terapéutico de las infecciones del tracto urinario en la infancia". An Pediatr (Barc) 2007; 67: $517-25$.

17.- Urinary tract infection in children: full guideline. 22 August 2007. http://guidance.nice. org.uk/CG054.

18.- Bradley J, Jackson MA and the Committee on Infectious Diseases. The use of systemic and topical fluoroquinolones. Pediatrics 2011; 128: e1034-45.

19.- Committee on Infectious Diseases. The use of systemic fluoroquinolones. Pediatrics 2006; 118: 1287-92.

20.- Peña A, Viviani T, Le Corre N, Morales V, Montecinos C, Gajardo C. Manejo de la infección del tracto urinario en lactantes febriles: Experiencia de tratamiento antimicrobiano intravenoso ambulatorio. Rev Chilena Infectol 2009; 26: 350-4.

21.- Shah G, Upadhyay J. Controversies in the diagnosis and management of urinary tract infections in children. Pediatr Drugs 2005; 7: $339-46$ 
22.- Hodson E M, Willis N S, Craig J C. Antibiotics for acute pyelonephritis in children. Cochrane Database Syst Rev 2007: CD003772.

23.- Cheng C H, Tsau Y K, Chen S Y, Lin T Y. Clinical courses of children with acute lobar nephronia correlated with computed tomographic patterns. Pediatr Infect Dis J 2009; 28: 300-3.

24.- Smaill F, Vásquez J C. Antibiotics for asymptomatic bacteriuria in pregnancy: Review. http://summaries.cochrane.org/CD000490/ antibiotics-for-asymptomatic-bacteriuria-inpregnancy

25.- Nicolle L E. Asymptomatic bacteriuria: review and discussion of the IDSA guidelines. Int $\mathrm{J}$ Antimicrob Agents 2006; 28 Suppl 1: S42-8.

26.- Doganis D, Siafas K, Mavrikou M, Issaris G, Martirosova A, Perperidis G, et al. Does early treatment of urinary tract infection prevent renal damage? Pediatrics 2007; 120: e922-8.

27.- Hewitt I K, Zuchetta P, Rigon L, Maschio P P, Molinari P P, Tomasi L, et al. Early treatment of acute pyelonephritis in children fails to reduce renal scarring: data from the Italian Renal Infection Study Trials. Pediatrics 2008; 122 : 486-90.

28.- Dayan P S, Hanson E, Bennett J E, Langsam D, Miller S Z. Clinical course of urinary tract infections in infants younger than 60 days of age. Pediatr Emerg Care 2004; 20: 85-8.

29.- Bachur R. Nonresponders: prolonged fever among infants with urinary tract infections. Pediatrics 2000; 105: e59-e62.

30.- Shaikh N, Ewing A, Bhatnagar S, Hoberman A. Risk of renal scarring in children with a first urinary tract infection: A systematic review. Pediatrics 2010; 126: 1084-91.

31.- Jodal U. The natural history of bacteriuria in childhood. Infect Dis Clin North Am 1987; 1: 713-29.

32.- Pennesi M, Travan L, Peratoner L, Bordugo A, Cattaneo A, Ronfani L, et al. Is antibiotic prophylaxis in children with vesicoureteral reflux effective in preventing pyelonephritis and renal scars? A randomized, controlled trial. Pediatrics 2008; 121: e1489-94.

33.- Garin E H, Olavarría F, García Nieto V, Valenciano B, Campos A, Young L. Clinical significance of primary vesicoureteral reflux and urinary antibiotic prophylaxis after acute pyelonephritis: a multicenter, randomized, controlled study. Pediatrics 2006; 117: 626-32.

34.- William G, Craig J C. Long-term antibiotics for preventing recurrent urinary tract infection in children. Cochrane Database Syst Rev 2011; (3): CD001534.

35.- Montini G, Hewitt I. Urinary tract infections: to prophylaxis or not to prophylaxis? Pediatr Nephrol 2009; 24: 1605-9.

36.- Salas P, Álvarez E, Saieh C. Pautas de diagnóstico y tratamiento en infección urinaria en niños. Documento de la Rama de Nefrología de la Sociedad Chilena de Pediatría. Rev Chil Pediatr 2003; 74: 311-14.

37.- Schoen E, Colby C, Ray G. Newborn circumcision decreases incidence and costs of urinary tract infections during the first year of life. Pediatrics 2000; 105: 789-93.

38.- Bauer R, Kogan B. New development in the diagnosis and management of pediatric UTIs. Urol Clin North Am 2008; 35: 47-53.

39.- Schoen E. Should newborns be circumcised? Can Fam Physician 2007; 53: 2096-8.

40.- Andres D. Should newborns be circumcised? Can Fam Physician 2007; 53: 2098-9.

41.- Jepson R, Craig J. Cranberries for preventing urinary tract infections. Cochrane Database Syst Rev 2008; (1): CD001321.

42.- Salo J, Uhari M, Helminen M, Korppi M, Nieminen T, Pokka T, et al. Cranberry juice for the prevention of recurrence of urinary tract infections in children: a randomized placebo-controlled trial. Clin Infect Dis 2012; 54: $340-46$.

43.- Mårild S, Hansson S, Jodal U, Odén A, Svedberg K. Protective effect of breast feeding against urinary tract infection. Acta Paediatr 2004; 93: 164-8.

44.- Levy I, Comarsca J, Davidovits M, Klinger G, Sirota L, Linder N. Urinary tract infection in preterm infants: the protective role of breast feeding. Pediatr Nephrol 2009; 24: 527-31.

45.- Girardin M, Seidman E G. Indications for the use of probiotics in gastrointestinal diseases. Dig Dis 2011; 29: 574-87.

46.- Abad C, Safdar N. The role of lactobacillus probiotics in the treatment or prevention of urogenital infections- a systematic review. J Chemother 2009; 21: 243-52.

47.- Williams G, Craig J C. Prevention of recurrent urinary tract infection in children. Curr Opin Infect Dis 2009; 22: 72-6.

48.- Lee S, Shim Y, Cho S, Lee J. Probiotics prophylaxis in children with persistent primary vesicoureteral reflux. Pediatr Nephrol 2007; 22: 1315-20.

49.- Storm D, Patel A, Koff S, Justice S. Novel management of urinary tract infections. Curr Opin Urol 2011; 21: 328-33.

50.- StormD, Koff S, Horvath D, Li B, Justice S. In vitro analysis of the bactericidal activity of Escherichia coli Nissle 1917 against pediatric uropathogens. J Urol 2011; 186 (4 Suppl): 1678-83.
51.- MontiniG, Tullus K, Hewitt I. Febrile urinary tract infections in children: N Engl J Med 2011; 365: $239-50$

52.- Tsung-Ning D, Huang F, Tsai T, Tsai J, Chiu N, Lin C. Clinical differentiation of acute pyelonephritis from lower urinary tract infection in children. J Microbiol Immunol Infect 2007; 40: 513-7.

53.- Leroy S, Gervaix A. Procalcitonin: a key marker in children with urinary tract infection. Adv Urol 2011; 2011: 397618.

54.- Kotoula A, Gardikis S, Tsalkidis A, Mantadakis E, Zissimopoulos A, Deftereos A, et al. Comparative efficacies of procalcitonin and conventional inflammatory markers for prediction of renal parenchymal inflammation in pediatric first urinary tract infection. Urology 2009; 73: 782-6.

55.- Cavagnaro F, Schonhaut L, Morales B, Espinoza A. Ecografía renal con Doppler en el diagnóstico de pielonefritis aguda en niños. Rev Chil Pediatr 2011; 82: 402-9.

56.- Majd M, Nussbaum Black A, Markle B, Shalaby-Rana E, Pohl H, Park J, et al. Acute pyelonephritis: Comparison of diagnosis with $99 \mathrm{mTc}$ - DMSA spect, spiral CT, MR imaging, and power Doppler US in an experimental pig model. Radiology 2001; 218: 101-8.

57.- Nanda N, Juthani-Mehta M. Novel biomarkers for the diagnosis of urinary tract infection-A systematic review. Biomark Insights 2009; 4: 111-21.

58.- Finnel S, Carroll AE, Downs S M; Subcommittee on Urinary Tract Infection. Technical report-Diagnosis and management of an initial UTI in febrile infants and young children. Pediatrics 2011; 128 (3): e749-70.

59.- Chertin B, Puri P. Familial vesicoureteral reflux. J Urol 2003; 169: 1804-8.

60.- Pohl H, Belman AB. The "top-down" approach to the evaluation of children with febrile urinary tract infections. Adv Urol 2009; 2009: 783409.

61.- Currie M, Mitz L, Raasch CS, Greenbaum L. Follow-up cultures and fever in children with urinary tract infection. Arch Pediatr Adolesc Med 2003; 157: 1237-40.

62.- American Academy of Pediatrics, Committee on Quality Improvement, Subcommittee on Urinary Tract Infection. Practice parameter: the diagnosis, treatment, and evaluation of the initial urinary tract infection in febrile infants and young children. Pediatrics 1999; 103: 84352.

63.- Tullus K. Difficulties in diagnosing urinary tract infections in small children. Pediatr Neprhrol 2011; 26: 1923-6. 